\title{
Assurance responsabilité civile professionnelle pour les médecins libres praticiens de Suisse: risque assurable ou état d'urgence?
}

\author{
Reinhard Kunz ${ }^{a}$, \\ Dr Markus Ackermann ${ }^{b}$ \\ a administrateur de FMH Services \\ b conseiller en gestion des risques
}

Sous l'impulsion d'informations et d'expériences rapportées par les sociétés médicales, FMH Services a chargé son administrateur, Monsieur Reinhard Kunz, de réaliser une étude sur l'évolution du marché des responsabilités civiles. Celui-ci a établi, en collaboration avec Monsieur Markus Ackermann, conseiller indépendant en gestion des risques, un rapport détaillé durant les mois de septembre à décembre 2005 en se basant essentiellement sur des entretiens menés avec les principaux assureurs suisses sur le thème de la responsabilité civile.

Dans le présent article, nous souhaitons vous informer brièvement sur l'évolution inhabituelle de cette branche d'assurance et sur les tendances observées.

\section{Situation actuelle dans le domaine de la responsabilité civile}

\section{Du point de vue du corps médical}

- Le renouvellement d'une police est souvent lié à de fortes augmentations de primes.

- Les disciplines médicales dont le succès thérapeutique relève fortement d'appréciations subjectives, telles que la «pure» chirurgie esthétique, n'obtiennent que très difficilement une couverture d'assurance.

- L'importance de la conclusion d'une assurance responsabilité civile pour couvrir le risque de l'entreprise «cabinet médical» va croissante.

- Depuis 1997, les membres de la FMH sont tenus par leur code de déontologie de contracter une assurance responsabilité civile professionnelle.

- L'obligation de conclure une telle assurance lors de l'ouverture de nouveaux cabinets médicaux existe, aujourd'hui déjà, dans 8 des 26 cantons.

- L'introduction générale de cette obligation fait actuellement l'objet de discussions soutenues.

- Les patients sont devenus plus critiques et plus exigeants face au succès d'un traitement.

- En sa qualité de client d'assurance, le médecin recherche des primes optimales et sa solidarité envers les disciplines médicales à haut risque tend à diminuer.

\section{Du point de vue des patients}

- Les patients sont mieux informés et s'intéressent davantage aux questions médicales.

- Leurs relations avec le médecin s'apparentent de plus en plus aux règles de la relation classique client-fournisseur de prestation, avec une sensibilité plus forte que naguère pour le résultat thérapeutique.

- Les organisations de patients, etc., encouragent les patients insatisfaits à engager des procédures.

- L'exigence accrue des patients s'exprime aussi dans une forte demande de dédommagements financiers.

- Les médias suisses et étrangers renforcent cette tendance.

- L'assurance de protection juridique largement répandue encourage et simplifie les procédures.

\section{Du point de vue des assureurs}

- La dérégulation du marché et la diminution des marges financières obligent les assureurs suisses à affiner leur évaluation des risques par branche d'assurance.

- La responsabilité civile est une branche placée sous le signe du long terme, c'est-à-dire qu'il se passe souvent de nombreuses années avant que l'étendue définitive des dommages soit connue et qu'un dossier puisse être clos.

- Les dommages sont souvent annoncés très tard à l'assureur. Au bout de cinq ans, il ne connaît que $50 \%$ des sinistres survenus.

- L'évolution déficitaire de cette branche d'assurance a été reconnue trop tard et les corrections nécessaires prennent aujourd'hui d'énormes proportions pour le corps médical.

- Le bilan est actuellement très déficitaire. Les indemnisations versées pour des sinistres dépassent en moyenne de 30\% les primes encaissées, c'est-à-dire que le taux de sinistres est supérieur à 130\%. Les indemnisations et les charges atteignent ensemble plus du 150\% des primes.

- Les assureurs témoignent d'un vif intérêt pour le corps médical en tant que groupe cible et cherchent des solutions acceptables pour les deux parties. 
- Se refusant à combler les déficits de l'assurance responsabilité civile professionnelle par des fonds en provenance d'autres branches d'assurance (subventionnement croisé), les assureurs réalisent l'assainissement nécessaire par des exclusions et des adaptations de primes.

- Alors que le nombre de sinistres ne change que peu par rapport au nombre de médecins, les cas de dommages dépassant 1,5 million de francs, rares autrefois, surviennent de plus en plus fréquemment.

- Les démarches juridiques à entreprendre pour protéger le médecin contre les revendications injustifiées constituent, aujourd'hui, un facteur important de coûts dans le traitement des sinistres.

- A quelques exceptions près, telles que la «pure» chirurgie esthétique, les assureurs restent intéressés à couvrir les risques inhérents à la responsabilité civile professionnelle pour toutes les disciplines médicales; ceci dans la mesure où les médecins acceptent les primes fixées.

\section{Tendances et évolutions pour demain}

- L'information des patients ira grandissant avec l'internet, les émissions médicales télévisées, etc.

- Les exigences actuelles des patients et leur tendance à revendiquer des indemnisations élevées tendent à rendre les cas de sinistres de plus en plus onéreux.

- Le renforcement du droit de la responsabilité civile en Suisse et dans les pays de l'Union européenne favorise cette évolution.

- Le nombre élevé d'assurances de protection juridique et l'extension des offres d'assurance, de même que la possibilité d'assurer des frais de procédure, favorisent les plaintes lors d'échecs thérapeutiques ce qui entraîne des coûts à long terme.

- Les assureurs limiteront ou excluront certains risques, par exemple pour des patients en provenance de pays dotés de droits renforcés en matière de responsabilité civile comme les Etats-Unis. Les mêmes patients demeurent toutefois couverts lorsque le traitement a lieu en Suisse en cas d'urgence.

- Les assureurs voudront prélever des primes en rapport avec les risques et assainir ou résilier les polices dont l'évolution est négative. Ces mauvais risques ne pourront être assurés qu'avec difficultés.

- Si les assureurs ne pouvaient pas gérer la responsabilité civile professionnelle de manière à couvrir les frais, certains d'entre eux se retireraient du marché.

- Les mêmes tendances se profilent dans les pays européens environnants.

En raison de cette évolution, l'assurance responsabilité civile se trouve dans le collimateur des assureurs et doit par conséquent être suivie avec attention.

\section{Recommandations}

Pour que la responsabilité civile professionnelle reste, à l'avenir, assurable pour la majorité des médecins, toutes les parties doivent participer de manière plus active à son évolution. Voici donc nos recommandations:

1. L'évolution de la responsabilité civile rend indispensable un dialogue régulier entre le corps médical (FMH / FMH Services) et les assureurs occupant une place de leader sur le marché. L'objectif visé est la transparence et la compréhension mutuelle.

2. Forts de cette transparence, il leur faut définir, développer et introduire ensemble des mesures propres à gérer les risques réels (gestion active des risques).

3. Le thème de la responsabilité civile sera redéfini et traité en profondeur dans l'offre de formation de FMH Services (ouverture de cabinet / reprise de cabinet).

4. Face à son assureur, chaque médecin peut continuer d'influencer fortement sa position individuelle si, outre la responsabilité civile, il conclut également avec lui d'autres polices d'assurance.

5. Concernant les disciplines particulières comme la «pure» chirurgie esthétique, des alternatives doivent être étudiées en Suisse et à l'étranger pour pallier à une éventuelle disparition de la couverture d'assurance dans ce champ très pointu.

\section{Résumé}

L'assurance responsabilité civile continuera à prendre de l'importance car il faut absolument couvrir ce type de risque, aujourd'hui comme demain. Actuellement, à l'exception de la «pure» chirurgie esthétique, il est erroné de parler d'une situation de détresse en matière d'assurance responsabilité civile. Les nouvelles augmentations de primes et/ou les limitations de couverture devront être tempérées ou évitées, ce qui nécessitera un dialogue actif entre le corps médical et les assureurs. L'évolution du marché des responsabilités professionnelles ne doit en aucun cas être abandonnée au hasard ou à elle-même. 